\title{
Cloud Monitoring Data Challenges: A Systematic Review
}

\author{
Asif Qumer Gill ${ }^{1}$ and Sarhang Hevary ${ }^{1}$, \\ ${ }^{1}$ University of Technology Sydney, School of Software \\ Ultimo NSW 2007, Australia \\ asif.gill@uts.edu.au, sarhang.hevary@alumni.uts.edu.au
}

\begin{abstract}
Organizations need to continuously monitor, source and process large amount of operational data for optimizing the cloud computing environment. The research problem is: what are cloud monitoring data challenges - in particular virtual CPU monitoring data? This paper adopts a Systematic Literature Review (SLR) approach to identify and report cloud monitoring data challenges. SLR approach was applied to initially identify a large set of 1861 papers. Finally, 24 of 1861 relevant papers were selected and reviewed to identify the five major challenges of cloud monitoring data: monitoring technology, virtualization technology, energy, availability and performance. The results of this review are expected to help researchers and practitioners to understand cloud computing data challenges and develop innovative techniques and strategies to deal with these challenges.
\end{abstract}

Keywords: Big Data, Cloud Computing, Capacity Planning, Monitoring, and Virtual CPU

\section{Introduction}

Cloud computing is a virtual data-intensive environment, which runs multiple virtual machines in large scalable clusters [11]. Cloud computing is one such new modes that supports pay-as-you-go and on-demand services (e.g. software as a service, platform as a service, infrastructure as a service) to enable business agility and flexibility [10]. Cloud computing seems to offer lucrative benefits [3], however, organizations need to actively monitor and analyze the operational data about the quality of cloud services and utilization of underlying virtual resources such as CPU, memory, storage and network [6]. This is also important to verify and identify any service performance related issues including Service Level Agreement (SLA). Monitoring is also important to track and control the expenses associated with the cloud service resource utilization [4].

There are a number of tools (e.g. AWS Cloud Watch) that claim to support the data monitoring including data acquisition and processing [e.g. 9]. However, to effectively adopt or develop specific cloud monitoring data tools, organizations need to identify and understand the fundamental challenges of cloud monitoring data. The understanding of challenges will help organizations in making informed decisions 
about the development and improvement of specific cloud monitoring data sourcing and processing tools for different types of cloud resources (e.g. CPU data, storage device data) at different levels (e.g. resource utilization data, health data). Cloud monitoring data is a broad topic. This paper mainly focuses on the monitoring challenges of virtual CPU utilization data within the overall context of cloud monitoring. Thus, the main research question is: what are cloud monitoring challenges - in particular virtual CPU utilization monitoring data?

This paper applied the well-known Systematic Literature Review (SLR) method [7] to systematically search, identify and synthesize the challenges of virtual CPU utilization monitoring data. This paper is organized as follows. Firstly, it discusses the research method. Secondly, it presents the research findings. Finally, it discusses the research findings and future research directions before concluding.

\section{Research Method}

This paper applied the SLR guidelines [7] for systematically searching, selecting, reviewing and synthesizing the cloud monitoring data challenges from relevant academic and industry publications (2011-2015). This study included the paper written in English language, which were selected from five well-known electronic databases (Table 3 ).

Table 1. Search keywords

\begin{tabular}{|c|c|}
\hline Search Category & Keywords/Phrases \\
\hline $\begin{array}{l}\text { Monitoring of Virtual CPU } \\
\text { (using advanced search } \\
\text { interfaces) }\end{array}$ & $\begin{array}{l}\text { Virtual Processor; Virtual CPU; Monitoring Tool; } \\
\text { Monitoring Technique; Cloud Computing; Virtual } \\
\text { Machine; SaaS Monitoring Technology. }\end{array}$ \\
\hline $\begin{array}{l}\text { Monitoring of Virtual CPU } \\
\text { (using advance command } \\
\text { search) }\end{array}$ & $\begin{array}{l}\text { (("virtual processor") OR ("virtual CPU")) AND } \\
((" \text { monitoring tool*") OR ("monitoring technique*")) } \\
\text { and (("cloud computing*")) } \\
\qquad((" * \text { virtual processor*") OR ("*virtual CPU*")) } \\
\text { AND (("*monitoring tool*") OR ("*monitoring } \\
\text { technique*")) and (("*cloud computing*")) }\end{array}$ \\
\hline
\end{tabular}

Table 1 presents the keyword or terms that were used during the first attempt to search the topic. All of the terms from the search category field "Monitoring of Virtual CPU" were also joined using the "AND" or "OR" operator to examine different combinations. In addition to this, search history features were used to combine different returned searched results to narrow down to the desired topics. Table 2 presents the paper selection criteria stages, which were applied to systematically identify the relevant papers for this study. 
Table 2. Paper selection criteria

\begin{tabular}{|c|c|c|}
\hline Filtration stage & Method & Assessment Criteria \\
\hline First Search Filtration & Explore the title & $\begin{array}{c}\text { Title }=\text { search keyword }(\mathbf{s}) \\
\text { Yes }=\text { accepted } \\
\text { No }=\text { rejected }\end{array}$ \\
\hline Second Search Filtration & Explore the abstract & $\begin{array}{l}\text { Abstract }=\text { CPU OR Virtual CPU } \\
\text { OR VCPU } \\
\text { Yes }=\text { accepted } \\
\text { No }=\text { rejected }\end{array}$ \\
\hline Final Search Filtration & Explore the content & $\begin{array}{ll}\text { 1. } & \begin{array}{l}\text { Address Virtual CPU OR } \\
\text { Monitoring Tool OR Cloud } \\
\text { Computing }\end{array} \\
\text { 2. } & \text { Well-referenced } \\
\text { 3. } & \text { Objective is clearly defined } \\
\text { 4. Well-presented argument and } & \text { justified } \\
\text { 5. Clearly stated findings } \\
\text { (Yes= accepted, No = rejected) }\end{array}$ \\
\hline
\end{tabular}

Table 3. Search results

\begin{tabular}{|l|l|l|l|}
\hline Database & 1st Search Filtration & 2nd Filtration & Final Count \\
\hline Web of Science & 462 & 40 & 3 \\
\hline IEEE & 913 & 30 & 10 \\
\hline Google Scholar & 67 & 16 & 7 \\
\hline Gartner & 12 & 6 & 2 \\
\hline Scopus & 407 & 5 & 2 \\
\hline Total & $\mathbf{1 8 6 1}$ & $\mathbf{9 7}$ & $\mathbf{2 4}$ \\
\hline
\end{tabular}

Initial search of keywords and filtration (based on title) across five selected databases resulted in a large number of 1861 papers. Second filtration resulted in 97 papers (based on the review of abstract), and finally, third filtration stage (based on the exploration of paper contents) resulted in 24 relevant papers for this review (Table 3 ). Please note that for the first and second search filtration stages, the items such as news, eBooks and tutorials were excluded as the contents were not suitable for this academic study. Finally, only those papers were selected that satisfied the five Assessment Criteria - Final Search Filtration (Table 2).

\section{Findings}

The selected 24 papers were analyzed and interpreted in order to answer the research question in hand. The detailed review of the selected papers resulted in five major challenge categories as shown in table 4 . These challenge categories are: monitoring technology, virtualization technology, energy, availability and performance. These categories were extracted after the careful review of the papers by using the analysis techniques from the well-known Grounded Theory [2], which is useful for identifying 
the relevant concepts and categories from a large volume of qualitative data or text [1]. Table 4 presents the identified major challenge categories and corresponding literature sources (see Appendix for selected literature sources S1-S24).

Table 4. Findings - data monitoring challenges categories

\begin{tabular}{|l|l|c|c|}
\hline Challenge Categories & Sources & $\begin{array}{l}\text { Frequency } \\
\text { (number } \\
\text { studies) }\end{array}$ & Percentage \\
\hline C1. Monitoring Technology & $\begin{array}{l}\text { S14, S15, S16, S17, } \\
\text { S18, S19, S20, S21 }\end{array}$ & 8 & $\mathbf{3 4 \%}$ \\
\hline C2. Virtualization Technology & S1, S2, S3, S4, S5, S6 & 6 & $\mathbf{2 5 \%}$ \\
\hline C3. Energy & S10, S11, S12, S13 & 4 & $\mathbf{1 7 \%}$ \\
\hline C4. Availability & S7, S8, S9 & 3 & $\mathbf{1 3 \%}$ \\
\hline C5. Performance & S22, S23, S24 & 3 & $\mathbf{1 3 \%}$ \\
\hline
\end{tabular}

\subsection{Monitoring Technology}

Monitoring of a specific remote virtual CPU resource is a challenge in the complex distributed cloud environment. Monitoring technology category is heavily referenced in the literature (e.g. $34 \%$ of the selected studies) and can help to resolve this challenge. (Table 4). This challenge category has identified three key underlying monitoring technology data challenges: (1) lack of data standardization, (2) live resource monitoring data, and (3) interoperability of data. Lack of monitoring data standards (e.g. templates, format) hinder the ability to integrate disparate monitoring tools and different types of virtual CPU data [e.g. S14]. The accurate monitoring data of a live virtual CPU resource in a cluster, which can be dynamically added, updated or removed, is challenging [e.g. S16]. Finally, third challenge is about the inability of the monitoring technology to support the interoperability of monitoring data across different cloud platforms [e.g. S18].

\subsection{Virtualization Technology}

Monitoring of the virtualized CPU resource data can also be impacted by the hypervisor, which is used to virtualize the physical CPU resource [5]. Interaction between the monitoring tool and hypervisor is important for collecting the utilization data. This is the second highly referenced category ( $25 \%$ of the selected studies) (Table 4). This challenge category has identified two key underlying challenges: (1) dual monitoring data and (2) heterogeneous virtual environment data. Hypervisor and virtualized CPU share physical resources and the challenge is that both need to be monitored for collecting the correct utilization data. Thus, this dual monitoring challenge needs to be addressed to accurately collect the utilization data [e.g. S1]. 
Heterogeneous virtual environment, containing different types of hypervisors and virtual CPUs, poses the challenge of dealing with different hypervisors' APIs and monitoring tools' APIs for monitoring, collecting and processing large amount of data in different formats [e.g. S2].

\subsection{Energy}

This is the third referenced category (17\% of the selected studies) (Table 4). This challenge category has identified two key underlying energy related challenges: (1) energy utilization data and (2) energy efficiency. Virtualized cloud environments claim to offer low energy utilization. These challenges draws our attention to the challenge of collecting and processing large amount of monitoring data using minimal energy or power. Energy utilization data needs to be monitored and optimized for energy efficiency [e.g. S10 and S11].

\subsection{Availability}

It is not about the CPU resource monitoring data. The monitoring should also provide the capability to collect and provide the virtual CPU availability data [e.g. S8]. This is the fourth referenced category (13\% of the selected studies) (Table 4). This challenge category has identified two key underlying availability related challenges: (1) SLA verification data, and (2) detecting and alerting data. The availability, in the context of virtual CPU utilization, is important and draws our attention to the challenge of monitoring availability SLAs, and then processing it for detecting and alerting any related issues or breaches [e.g. S7 and S8].

\subsection{Performance}

Finally, this category draws our attention to the computational performance challenge of both the virtual machines and monitoring. This is the fifth referenced category (13\% of the selected studies) (Table 4). This challenge category has identified two key challenges: (1) performance identification, and (2) detecting and alerting. The monitoring should provide the capability to collect and provide the data about the performance of the virtual CPU resource and monitoring technology to help detecting and alerting any performance issues [e.g. S24]. Based on the performance results, we can dynamically adjust the utilization and control the number of virtual CPUs assigned to a physical CPU [e.g. S24]. Further, performance results can lead to the consolidation and de-consolidation of the virtual CPUs and underlying physical resources [e.g. S22 and S23]. 


\section{Discussion}

The effective utilization of cloud requires monitoring the hypervisors and the virtual environments. Monitoring data growth and velocity are increasing, and different monitoring standards, architectures, tools, and APIs are required to monitor the resource usage and capture a large amount of operational data [8]. However, the monitoring of cloud, in particular virtual CPUs hosted on a heterogeneous environment, poses several challenges. This paper addresses this research problem and systematically identify the five key challenges categories and underlying challenges.

Firstly, our findings highlighted that monitoring (34\%) and virtualization (24\%) technology were the most important challenge categories with respect to virtual CPU monitoring data collection and processing. Thus, we can classify these two as core challenge categories. Other challenge categories such as energy (17\%), availability $(13 \%)$, and performance $(13 \%)$ were classified as secondary. This is because they were not heavily reported, although, they could impact the monitoring (Table 4).

Secondly, our findings highlighted that the effective monitoring of the heterogeneous environment requires monitoring standards and frameworks for monitoring data integration and interoperability across different cloud platforms. This is important to facilitate the effective adoption of cloud.

Thirdly, our findings highlighted that the capturing and processing of the monitoring data are not enough. Monitoring capability should also support detecting any issues and alerting or taking corrective actions or adjustments. This leads to the identification of whole new area of research about smart data-driven and analyticsenabled adaptive monitoring. Monitoring of large and complex environment generates huge amount of data, which draws our attention to another area of research about BigData analytics for cloud monitoring data.

Similar to any other SLR studies, this paper has some limitations. One limitation could be the use of finite number of selected literature databases and studies. This paper included studies from well-known databases, and we have full confidence that the selected databases and studies provided us with the relevant and recent literature to address the research question in hand. One may argue about the possible bias in the selection of studies and inaccuracy of analysis. To mitigate this risk, we developed and applied relevant search string and keywords, systematic study selection criteria (Tables 1-2) and analysis techniques from well-known Grounded Theory [2]. This was done to ensure that the relevant studies were not omitted.

Despite possible limitations, this paper provided useful insights for both practitioners and researchers interested in the area of cloud monitoring data capturing and processing. For instance, practitioners may be interested in developing new tools, formats and standards for exchanging monitoring data across different cloud platforms. Researchers may be interested in developing new frameworks for BigData analytics enabled smart and adaptive monitoring. 


\section{Conclusion}

This paper presented a SLR of virtual CPU utilization monitoring data challenges. This paper systematically searched, identified and reviewed a set of twenty-four relevant papers. The detailed review of selected papers provided us with the five major challenge categories. This study provided a knowledge-base of monitoring data challenges to practitioners and researchers who have interest in cloud computing. The findings of this paper can be further used in developing monitoring data sharing and processing standards, formats and tools to facilitate the effective cloud monitoring data management. The findings of this paper will provide necessary inputs to further research and develop the BigData analytics enabled framework for smart and adaptive cloud monitoring data.

\section{References}

1. Alzoubi, Y. I., Gill, A. Q., \& Al-Ani, A. Empirical studies of geographically distributed agile development communication challenges: A systematic review. Information \& Management, 53(1), 22-37, (2016).

2. GTI, what is Grounded Theory? (2008). http://www.groundedtheory.com/what-is-gt.aspx.

3. Gill, A. Q., Bunker, D., \& Seltsikas, P. Moving Forward: Emerging Themes in Financial Services Technologies' Adoption. Communications of the Association for Information Systems, 36, (2015).

4. Gill, A. Q. Adaptive Cloud Enterprise Architecture. World Scientific, (2015).

5. Gill, A. Q., Bunker, D., \& Seltsikas, P. An empirical analysis of cloud, mobile, social and green computing: Financial services it strategy and enterprise architecture. In Dependable, Autonomic and Secure Computing (DASC), 2011 IEEE Ninth International Conference on (pp. 697-704). IEEE, (2011).

6. Jamail, N. S. M., Atan, R., Abdullah, R., \& Said, M. Y. Development of SLA Monitoring Tools Based on Proposed DMI in Cloud Computing. Transactions on Machine Learning and Artificial Intelligence, 3(1), 01, (2015).

7. Kitchenham, B. A., \& Charters, S. (2007). Procedures for performing systematic literature reviews in software engineering. Keele University \& Durham University, UK, (2007).

8. Kowall, J \& Fletcher C, 2014, Modernize Your Monitoring Strategy by Combining Unified Monitoring and Log Analytics Tools, (2014). Gartner http://www.gartner.com/document/code/257830?ref=grbody\&refval=2809724

9. Meng, S., \& Liu, L. Enhanced monitoring-as-a-service for effective cloud management. Computers, IEEE Transactions on, 62(9), 1705-1720, (2013).

10. NIST (2011). NIST Cloud Computing Reference Architecture. http://www.nist.gov/customcf/get_pdf.cfm?pub_id=909505.

11. Smith, S., Gill, A. Q., Hasan, H., \& Ghobadi, S. An Enterprise Architecture Driven Approach to Virtualisation. PACIS 2013 Proceedings, (2013).

\section{Appendix: Selected papers included in this review}

[S1] Anand, Ankit, et al. "Resource usage monitoring for KVM based virtual machines." Advanced Computing and Communications (ADCOM), 2012 18th Annual International Conference on. IEEE, 2012. 
[S2] Du, Jiaqing, Nipun Sehrawat, and Willy Zwaenepoel. "Performance profiling of virtual machines." ACM SIGPLAN Notices 46.7 (2011): 3-14.

[S3] P. Vijaya Vardhan Reddy, P. Vijaya Vardhan Reddy, and Dr Lakshmi Rajamani Dr. Lakshmi Rajamani. "Performance Evaluation of Hypervisors in the Private Cloud based on System Information using SIGAR Framework and for System Workloads using Passmark." International Journal of Advanced Science and Technology 70 (2014): 17-32

[S4] Reddy, P., and Lakshmi Rajamani. "Performance comparison of different operating systems in the private cloud with KVM hypervisor using SIGAR framework." Communication, Information \& Computing Technology (ICCICT), 2015 International Conference on. IEEE, 2015

[S5] Reddy, P. Vijaya Vardhan, and Lakshmi Rajamani. "Evaluation of Different Hypervisors Performance in the Private Cloud with SIGAR Framework."International Journal of Advanced Computer Science and Applications (IJACSA) 5.2 (2014).

[S6] Reddy, P., and Lakshmi Rajamani. "Virtualization overhead findings of four hypervisors in the CloudStack with SIGAR." Information and Communication Technologies (WICT), 2014 Fourth World Congress on. IEEE, 2014.

[S7] Houlihan, Ryan, et al. "Auditing cloud service level agreement on VM CPU speed." Communications (ICC), 2014 IEEE International Conference on. IEEE, 2014

[S8] Huang, Qiang, et al. "Auditing CPU Performance in Public Cloud." Services (SERVICES), 2013 IEEE Ninth World Congress on. IEEE, 2013.

[S9] Alhamazani, Khalid, et al. "An overview of the commercial cloud monitoring tools: research dimensions, design issues, and state-of-the-art." Computing (2014): 1-21.

[S10] Madani, N., et al. "Power-aware Virtual Machines consolidation architecture based on CPU load scheduling." Computer Systems and Applications (AICCSA), 2014 IEEE/ACS 11th International Conference on. IEEE, 2014.

[S11] Vrbsky, Susan V., et al. "Decreasing power consumption with energy efficient data aware strategies." Future Generation Computer Systems 29.5 (2013): 1152-1163

[S12] Katsaros, Gregory, et al. "A service framework for energy-aware monitoring and VM management in Clouds." Future Generation Computer Systems 29.8 (2013): 2077-2091.

[S13] Verma, Akshat, Puneet Ahuja, and Anindya Neogi. "pMapper: power and migration cost aware application placement in virtualized systems." Middleware 2008. Springer Berlin Heidelberg, 2008. 243264.

[S14] Katsaros, Gregory, et al. "A Self-adaptive hierarchical monitoring mechanism for Clouds." Journal of Systems and Software 85.5 (2012): 1029-1041

[S15] Povedano-Molina, Javier, et al. "DARGOS: A highly adaptable and scalable monitoring architecture for multi-tenant Clouds." Future Generation Computer Systems 29.8 (2013): 2041-2056.

[S16] Smit, Michael, Bradley Simmons, and Marin Litoiu. "Distributed, application-level monitoring for heterogeneous clouds using stream processing." Future Generation Computer Systems 29.8 (2013): 2103 2114.

[S17] Lee, Hyungro, et al. "Towards Understanding Cloud Usage through Resource Allocation Analysis on XSEDE."

[S18] Ranjan, Rajiv, et al. "A note on software tools and techniques for monitoring and prediction of cloud services." Software: Practice and Experience 44.7 (2014): 771-775.

[S19] Serrano, Nicolas, Gorka Gallardo, and Josune Hernantes. "Infrastructure as a Service and Cloud Technologies." IEEE Software 2 (2015): 30-36.

[S20] Manvi, Sunilkumar S., and Gopal Krishna Shyam. "Resource management for Infrastructure as a Service (IaaS) in cloud computing: A survey." Journal of Network and Computer Applications 41 (2014): 424-440.

[S21] Dhingra, Mohit, J. Lakshmi, and S. K. Nandy. "Resource usage monitoring in clouds." Proceedings of the $2012 \mathrm{ACM} / \mathrm{IEEE}$ 13th International Conference on Grid Computing. IEEE Computer Society, 2012.

[S22] Reddy, P., and Lakshmi Rajamani. "Performance comparison of different operating systems in the private cloud with KVM hypervisor using SIGAR framework." Communication, Information \& Computing Technology (ICCICT), 2015 International Conference on. IEEE, 2015

[S23] Janpan, Tanasak, Vasaka Visoottiviseth, and Ryousei Takano. "A virtual machine consolidation framework for CloudStack platforms." Information Networking (ICOIN), 2014 International Conference on. IEEE, 2014.

[S24] Miao, Tianxiang, and Haibo Chen. "FlexCore: Dynamic virtual machine scheduling using VCPU ballooning." Tsinghua Science and Technology 20.1 (2015): 7-16. 